\title{
Study of impact of COVID-19 infection on ectopic pregnancy in a tertiary care center
}

\author{
Niranjan N. Chavan, Hitendrasing D. Rajput*, Dinesh Wade, Shikhanshi
}

Department of Obstetrics and Gynecology, LTMMC and LTMGH, Sion, Mumbai, Maharashtra, India

Received: 01 February 2021

Revised: 09 March 2021

Accepted: 10 March 2021

\author{
*Correspondence: \\ Dr. Hitendrasing D. Rajput, \\ E-mail: hitendra193@gmail.com
}

Copyright: () the author(s), publisher and licensee Medip Academy. This is an open-access article distributed under the terms of the Creative Commons Attribution Non-Commercial License, which permits unrestricted non-commercial use, distribution, and reproduction in any medium, provided the original work is properly cited.

\begin{abstract}
Background: COVID-19 is an infectious disease caused by a recently discovered coronavirus (SARS-CoV-2). Most of the people infected with the coronavirus experience mild to moderate respiratory illness and recover without requiring any special treatment. In current study we have studied impact of COVID-19 pandemic on clinical outcome and management of ectopic pregnancy.

Methods: Women with confirmed or suspected ectopic pregnancy admitted in emergency for further management and tested via nasopharyngeal (NP) or oropharyngeal swab for SARS-CoV-2 using reverse transcriptase polymerase chain reaction (RT-PCR) from April 1, 2020 to November 30, 2020 were included in the study.

Results: In our study out of 32 case of ectopic pregnancy, 3 patients were COVID-19 positive. Total non COVID-19 patients were 29 . Out of 32 ectopic pregnancies only 4 were unruptured ectopic pregnancy and rest 28 had ruptured ectopic pregnancy. Mortality among ectopic pregnancy was noted only in 1 case $(3.12 \%)$.

Conclusions: Timely diagnosis and initiating management at first point of care can reduce the morbidity and mortality due to ectopic pregnancy. Patient's knowledge attitude and awareness will be achieved through health education. Women with clinical signs and physical symptoms of a ruptured ectopic pregnancy, such as hemodynamic instability or an acute abdomen, should be evaluated and treated urgently. In pandemic situation where some part of hospital facilities devoted to COVID-19 patients, so planned distribution of resources to tackle medical emergency will bear fruitful positive outcome.
\end{abstract}

Keywords: COVID-19, Coronavirus, Fever, Maternal morbidity, Maternal mortality, SARS-CoV-2

\section{INTRODUCTION}

COVID-19 is an infectious disease caused by a recently discovered coronavirus (SARS-CoV-2). Most of the people infected with the coronavirus will experience mild to moderate respiratory illness and recover without requiring any special treatment. Elderly people and those with underlying medical problems like cardiovascular disease, diabetes, chronic respiratory disease, any chronic illness and malignancies are more likely to develop serious illness and related complications. Implantation of fertilized ovum at site other than normal uterine cavity is known as an ectopic pregnancy. ${ }^{1}$ Ectopic Pregnancy is a life threatening emergency associated with significant mortality. ${ }^{1}$ Routinely diagnosis shall start not only with clinical suspicion but also needs to be confirmed with supportive evidences from radiological findings which help in deciding further line of management. ${ }^{2}$ Female of reproductive age group who are presenting with complaints of lower abdominal pain or vaginal bleeding with missed period will rise strong suspicion of an ectopic pregnancy. ${ }^{1,3,4}$ Early diagnosis and prompt management helps in reduction of not only the mortality but also morbidity of condition can be reduced. In current study we have studied impact COVID-19 pandemic on clinical outcome and management of ectopic pregnancy. 
Major cause of maternal morbidity and mortality are studied, also medical and surgical option of treatment modalities are compared to frame best management strategy for better outcome during the adversity of COVID-19 pandemic time.

\section{Aims and objectives}

Aims and objectives were to study impact of COVID-19 pandemic on ectopic pregnancy and to study factors contributing maternal morbidity and mortality in ectopic pregnancy.

\section{METHODS}

\section{Study design}

This study was conducted at Lokamanya Tilak Municipal Medical College and General Hospital (Mumbai, India), a tertiary care referral hospital and currently leading in management of COVID-19 pandemic.

Women with confirmed or suspected ectopic pregnancy admitted in emergency for further management and tested via nasopharyngeal (NP) or oropharyngeal swab for SARS-CoV-2 using reverse transcriptase polymerase chain reaction (RT-PCR) from April 1, 2020 to November 30, 2020 were included in this study. Total 32 cases of ectopic pregnancy were studied during this study period.

\section{Parameters studied}

ANC registration, Referral status, Signs and symptoms of ectopic pregnancy and COVID-19 infection, obstetrics history, past history of abdominal and pelvic surgeries, history of abortions, history of pelvic inflammatory infections, type of ectopic pregnancy, need for blood transfusion, need for ventilator support and management given.

\section{Statistical analysis}

All the parameters were studied and analysed on the basis of percentages. As this was a purely observational study, the maternal parameters were analysed using descriptive statistics i.e. percentages and proportions were calculated and no statistical test was applied.

\section{RESULTS}

Total ectopic pregnancy cases were 32. Total COVID-19 positive cases were 3 .

Table 1: Distribution of cases $(n=32)$.

\begin{tabular}{|lll|}
\hline Cases & Number & Percentage \\
\hline COVID-19 Positive & 3 & 9.37 \\
\hline Non COVID-19 cases & 29 & 90.67 \\
\hline
\end{tabular}

Table 1: Out of 32 case of ectopic pregnancy, 3 patients were COVID-19 positive and total non COVID-19 patients were 29 .

Table 2: Distribution according to ANC registration $(n=32)$.

\begin{tabular}{|lll|}
\hline Cases & Number & Percentage \\
\hline Registered & 2 & 6.25 \\
\hline Unregistered & 30 & 93.75 \\
\hline
\end{tabular}

Table 2: Out of 32 patients, only 2 patients were ANC registered and rest 30 were unregistered.

Table 3: Distribution according to referral status $(n=32)$.

\begin{tabular}{|lll|}
\hline Cases & Number & Percentage \\
\hline Referred & 9 & 28.12 \\
\hline $\begin{array}{l}\text { Direct emergency } \\
\text { admissions }\end{array}$ & 23 & 71.87 \\
\hline
\end{tabular}

Table 3: Out of 32 patients, 9 were referred from periphery hospitals due to non-availability of ICU facilities and rest 23 patients directly visited to emergency room.

Table 4: Distribution of cases according to classical triad of ectopic pregnancy $(n=32)$.

\begin{tabular}{|lll|}
\hline Cases & Number & Percentage \\
\hline Classical triad & 20 & 62.5 \\
\hline No classical triad & 12 & 37.5 \\
\hline
\end{tabular}

Table 4: Ectopic pregnancy characterised by classical triad of pain in abdomen, PV bleeding and amenorrhea. But this classical triad was noted in only 20 patients $(62.5 \%)$.

Table 5: Distribution according to gravida status $(n=32)$.

\begin{tabular}{|lll|}
\hline Cases & Number & Percentage \\
\hline Primigravida & 3 & 9.37 \\
\hline Multigravida & 29 & 90.62 \\
\hline
\end{tabular}

Table 5: 3 patients were primigravida and 29 were multigravida status.

Table 6: Distribution of according to previous pelvic surgeries $(n=32)$.

\begin{tabular}{|lll|}
\hline Cases & Number & Percentage \\
\hline Yes & 2 & 6.25 \\
\hline No & 30 & 93.75 \\
\hline
\end{tabular}

Table 6: 2 cases had history of previous pelvic surgery which was considered as contributory factor for ectopic pregnancy. 
Table 7: Distribution of according to previous abdominal surgeries $(n=32)$.

\begin{tabular}{|lll|}
\hline Cases & Number & Percentage \\
\hline Yes & 3 & 9.37 \\
\hline No & 29 & 90.62 \\
\hline
\end{tabular}

Table 7: 3 patients had previous abdominal surgery.

Table 8: Distribution according to history of PID $(n=32)$.

\begin{tabular}{|lll|}
\hline Cases & Number & Percentage \\
\hline Yes & 3 & 9.37 \\
\hline No & 29 & 90.62 \\
\hline
\end{tabular}

Table 8: 3 patients had history of previously diagnosed PID.

Table 9: Distribution according to types of ectopic pregnancy $(\mathbf{n = 3 2})$.

\begin{tabular}{|lll|}
\hline Cases & Number & Percentage \\
\hline Tubal & 31 & 96.87 \\
\hline Ovarian & 1 & 3.12 \\
\hline
\end{tabular}

Table 9: Among ectopic pregnancies 31 patients had tubal ectopic and 1 had ovarian ectopic pregnancy. During study period no case of cervical, abdominal and scar ectopic found.

Equal number of cases of right (16 cases) and left sided (16 cases) ectopic were noted. Out of 32 ectopic pregnancies only 4 were unruptured ectopic pregnancy and rest 28 had ruptured ectopic pregnancy and history of abortion was noted in 8 cases.

Though 3 patients were COVID-19 positive but no signs and symptoms were found due to infection. On evaluation USG was most common modality to confirm the diagnosis of ectopic pregnancy.

All 32 cases were managed by exploratory laparotomy out of which 10 patients required ventilatory support in post op period and 20 patients of ectopic pregnancy required blood transfusion. Mortality among ectopic pregnancy in our study was noted only in 1 case $(3.12 \%)$ and this patient was COVID-19 positive and cause of death was cardiac arrest secondary to haemorrhagic shock because of ruptured tubal ectopic pregnancy.

\section{DISCUSSION}

Ectopic pregnancy is a common problem, occurring in about 11 in 1000 pregnancies. ${ }^{3}$ It is a leading cause of maternal mortality in the first trimester of pregnancy which is responsible for $0.2 \%$ of total maternal mortality. Important key for diagnosis is to look for the presence or absence of an intrauterine gestational sac and then it has to be correlated with quantitative serum beta hCG (ß$\mathrm{hCG}$ ) levels. Ectopic pregnancy is any pregnancy in which the fertilized ovum gets implanted outside the intrauterine cavity. More than 95 percent of ectopic pregnancies occur in the fallopian tubes and in fallopian tube most common location is ampulla of fallopian tube. ${ }^{1}$ Another 2.5 percent occur in the cornua of the uterus, and the remaining are noted in the cervix, ovary or abdominal cavity. Because none of these anatomic sites can easily accommodate placental attachment or a growing embryo, the potential risk for rupture and haemorrhage always exists in all types of ectopic pregnancy. Many women may not be aware of an ongoing pregnancy and hence may not anticipate a pregnancy related complication. Such women are most often seen first with complaints of pain in abdomen by a general practitioner and hence, the importance of careful history taking along with patient education cannot be overemphasised.

Risk factors which are associated with ectopic pregnancy have a common mechanism of action namely, interference with functions of fallopian tube. Normally, an egg gets fertilized in the ampulla of fallopian tube and then travels down the tube to gate implanted in endometrium. Any mechanism which interferes with the normal function of the fallopian tube during this process increases the risk of abnormal implantation at unwanted site and leads to ectopic pregnancy. Previous history of ectopic pregnancy still remains a more significant risk factor, with each successive occurrence. With one previous ectopic pregnancy treated have an increased risk of ectopic pregnancy, although an intervening intrauterine pregnancy lowers this rate.

Early diagnosis, identifying of underlying risk factors and timely intervention in the form of conservative or surgical treatment will help in reducing the significant morbidity and mortality associated with ectopic pregnancy. ${ }^{2}$ Endosalpingitis damages the mucosa and may cause an entrapment of the migrating embryo, leading to unwanted implantation. Exosalpingitis leads peritubal adhesion along with impairing peristaltic movements of tubes which is the reason for inadequate transportation of fertilized embryo in uterine cavity. ${ }^{2}$

The commonest presenting complaints are abdominal pain, amenorrhea and abnormal vaginal bleeding. ${ }^{2}$ Studies have shown that Ultrasonography should be the first investigation for symptomatic women in their first trimester for early detection of ectopic pregnancy. ${ }^{2}$ The commonest site of location of the ectopic pregnancy is in the ampulla of the fallopian tube..$^{2,5,1,6}$ Early diagnosis is aided by a high index of suspicion. Every sexually active, reproductive aged woman who presents with pain in abdomen or abnormal vaginal bleeding should be screened for pregnancy, regardless of whether she is currently using contraception or not. ${ }^{1,3,4,6}$ Women may present with nonspecific symptoms, unaware of an ongoing pregnancy or even present with haemodynamic shock. ${ }^{6}$ 
In many of the underdeveloped countries, majority of patients are still diagnosed after tubal rupture. In Our centre being a tertiary level referral centre $86.95 \%$ cases came with ruptured ectopic and most common reason for this is either late referrals from other hospitals or delay in seeking medical help because of ongoing COVID-19 pandemic. $^{6}$

Among the risk factors in our study we found out, previous pelvic surgery was the most common, followed by previous abortions. ${ }^{6}$ Although clinical examination may raise suspicion of an ectopic pregnancy, but relying only on clinical signs and symptoms alone will missed the diagnosis in majority of cases and will also increase the risk of mortality and morbidity. ${ }^{6}$ Ultrasonography was useful in diagnosing most of these cases, with the exception in one case, in which we directly went for laparotomy for diagnosis and immediate management as patient was hemodynamically unstable and was having strong clinical suspicion of ruptured ectopic pregnancy. Prevention and treatment of PID and encouraging women to undergo an early transvaginal ultrasonography to confirm the location of pregnancy is likely to prevent late diagnosis and adverse outcome and will also reduce significant amount of mortality. ${ }^{6}$

As medical management needs extremely close follow up and hospitalization, surgical management is still the method of choice in our country. ${ }^{2,7}$ Medical treatment of ectopic pregnancy with methotrexate is an increasingly common alternative to surgical management because of very low incidence of complications. ${ }^{8}$ Surgical management of ectopic pregnancy is required when a patient is exhibiting any of the following: hemodynamic instability, symptoms of an ongoing ruptured ectopic or signs of hemoperitoneum. ${ }^{1}$ Majority of cases of ectopic pregnancy still managed better by laparotomy. ${ }^{6}$ Whereas in our study we also managed all 32 cases by laparotomy. ${ }^{6}$ As many of them presented with ruptured ectopic.

The novel coronavirus infection (COVID-19) is a recent global public health emergency and the mode of transmission is by droplets spread which can occur when the patient sneezes or coughs. The incubation period varies from 2 days to 2 weeks following exposure to the virus. ${ }^{9}$ The patient can present with many symptoms, most commonly presenting with complaints of cold, cough, fever, headache, malaise, weakness, flushing of face, itching or watering in the eyes. However, patients may also present with gradual worsening of respiratory discomfort or multi organ failure in late stages. The diagnosis of current infection depends on tests to detect the presence of virus in respiratory tract secretions. The standard test being done presently is detection of the viral RNA by RT-PCR (Reverse Transcriptase Polymerase Chain Reaction) from the nasopharyngeal mucosa or oropharyngeal mucosa as recommended by the ICMR (Indian Council of Medical Research). ${ }^{9}$
Genetic analysis of COVID-19 virus revealed that it is similar to severe acute respiratory syndrome coronavirus (SARS-CoV- 2). Unlike SARS-CoV-2, transmission of COVID-19 occurs during the prodromal period when those infected are mildly ill, and carry on usual activities, which contributes to the spread of infection. According to the report on "Diamond Princess", among the 1,723 tested travellers, 189 asymptomatic individuals were positive for the COVID-19 virus as of 17 February 2020 (Wikipedia, 2020), which indicated that a very large number of asymptomatic carriers and mild patients remain undiagnosed in the community. ${ }^{10,11}$ In our study we had total 3 cases of COVID-19 and all three were asymptomatic for COVID-19. It is crucial to identify and isolate asymptomatic carriers and mild patients in order to limit the outbreaks in later stages. ${ }^{10}$

Effect of COVID-19 on ectopic pregnancy - Related to our study we have seen majority of ectopic patient presented as ruptured ectopic which may be due to delay in seeking hospital care due to ongoing COVID-19 pandemic and its consequences like lockdown, travelling issues, less availability of private hospitals and fear of getting COVID-19 infection.

Limitations: Though study is about ectopic and its management in COVID-19 pandemic situation but for emergency situations guidelines and management protocol were still as previous to pandemic time. Only confounding factor of approach to medical services was unpredictable and dependent on other environmental and cultural factors which exist unevenly pre and post pandemic time.

\section{CONCLUSION}

Ectopic pregnancy is a significant cause of maternal mortality and morbidity. Though its incidence is increasing but mortality has reduced considerably due to advances in early diagnosis by USG and Beta HCG assays. In our study all the cases were diagnosed with a higher clinical suspicion and the USG findings helped to confirm the diagnosis. Though the recent trend in the management of ectopic pregnancy is the use of a conservative surgical or medical line of management but in our study salpingectomy was the treatment modality which was used. This was mainly because majority $(80 \%)$ of the cases were transferred from other hospitals due to non-availability of ICU facilities and hence came late to our hospital after the ectopic pregnancy has ruptured. It is pertinent to note that in lockdown pandemic situation, there were restricted transport facilities. Extra workload of COVID-19 pandemic adds to extra delay in transferring patient to a tertiary care health facility as well as there was also a delay from patients side, in seeking medical help due to fear of contracting COVID-19 infection.

Timely diagnosis and initiating management at first point of care can reduce the morbidity and mortality due to 
ectopic pregnancy. In conclusion, identifying undiagnosed underlying risk factors of ectopic pregnancy, availability of adequate health care facilities, timely ultrasound examination along with availability of beta hCG assay and in time intervention will help reduce the morbidity and mortality due to ectopic pregnancy. Early treatment of pelvic infections will also be a useful preventive measures and screening for the same will add on to decrease ectopic incidence rate. Every sexually active, reproductive aged female who comes with abdominal pain or bleeding from vagina should be screened for pregnancy and confirmation of location of pregnancy, regardless of whether she is currently using contraception. Patient's knowledge attitude and awareness will be achieved through health education. Women with clinical signs and physical symptoms of a ruptured ectopic pregnancy, such as hemodynamic instability or an acute abdomen, should be evaluated and treated urgently.

In pandemic situation where some part of hospital facilities devoted to COVID-19 patients so planned distribution of resources to tackle medical emergency will bear fruitful positive outcome.

Funding: No funding sources Conflict of interest: None declared

Ethical approval: Not required

\section{REFERENCES}

1. Pregnancy TE. ACOG Practice Bulletin No. 191: Tubal Ectopic Pregnancy. Obstet Gynecol. 2018;131(2):e65-77.

2. Sudha V, Thangaraj D. A retrospective study on ectopic pregnancy: a two year study. Int $\mathrm{J}$ Reprod Contraception, Obstet Gynecol. 2016;5(12):4365-8.

3. Kirk E, Papageorghiou AT, Condous G, Tan L, Bora $\mathrm{S}$, Bourne $\mathrm{T}$. The diagnostic effectiveness of an initial transvaginal scan in detecting ectopic pregnancy. Hum Reprod. 2007;22(11):2824-8.

4. van Mello NM, Mol F, Opmeer BC, Ankum WM, Barnhart K, Coomarasamy A, et al. Diagnostic value of serum hCG on the outcome of pregnancy of unknown location: A systematic review and metaanalysis. Hum Reprod Update. 2012;18(6):603-17.

5. Swende TZ, Jogo AA. Ruptured tubal pregnancy in Makurdi, north central Nigeria. Niger J Med. 2008;17(1):75-7.

6. Tahmina S, Daniel M, Solomon P. Clinical analysis of ectopic pregnancies in a tertiary care centre in Southern India: A six-year retrospective study. J Clin Diagnostic Res. 2016;10(10):QC13-6.

7. Chatterjee S, Dey S, Chowdhury RG. Ectopic Pregnancy In Previously Infertile Women Subsequent Pregnancy Outcome After Laparoscopic Management. 2009;2:67-72.

8. Isaacs R, Mcgehee RP, Cowan BD. Treatment Pregnancy : Cases of Ectopic Report. 50(96):694-6.

9. Nayak AH, Kapote DS, Fonseca M, Chavan N, Mayekar R, Sarmalkar M, et al. Impact of the Coronavirus Infection in Pregnancy: A Preliminary Study of 141 Patients. J Obstet Gynecol India 2020;70(4):256-61.

10. Hu Z, Song C, Xu C, Jin G, Chen Y, Xu X, et al. 11427_2020_Article_1661.2020;

11. Liu Y, Song Y, Hu X, Yan L, Zhu X. Awareness of surgical smoke hazards and enhancement of surgical smoke prevention among the gynecologists. J Cancer. 2019;10(12):2788-99.

Cite this article as: Chavan NN, Rajput HD, Wade D, Shikhanshi. Study of impact of COVID-19 infection on ectopic pregnancy in a tertiary care center. Int J Reprod Contracept Obstet Gynecol 2021;10:1660-4. 